\title{
On Finding Separators in Temporal Split and Permutation Graphs*
}

\author{
Nicolas Maack ${ }^{1}$, Hendrik Molter ${ }^{2}$, Rolf Niedermeier ${ }^{1}$, and Malte Renken ${ }^{1}$ \\ ${ }^{1} \mathrm{TU}$ Berlin, Algorithmics and Computational Complexity, Berlin, Germany, \\ nicolas.km.maack@campus.tu-berlin.de, \\ \{rolf.niedermeier, m.renken\}@tu-berlin.de \\ ${ }^{2}$ Department of Industrial Engineering and Management, \\ Ben-Gurion University of the Negev, Beer-Sheva, Israel, molterh@post.bgu.ac.il
}

\begin{abstract}
Removing all connections between two vertices $s$ and $z$ in a graph by removing a minimum number of vertices is a fundamental problem in algorithmic graph theory. This $(s, z)$ separation problem is well-known to be polynomial solvable and serves as an important primitive in many applications related to network connectivity.

We study the NP-hard temporal $(s, z)$-separation problem on temporal graphs, which are graphs with fixed vertex sets but edge sets that change over discrete time steps. We tackle this problem by restricting the layers (i.e., graphs characterized by edges that are present at a certain point in time) to specific graph classes.

We restrict the layers of the temporal graphs to be either all split graphs or all permutation graphs (both being perfect graph classes) and provide both intractability and tractability results. In particular, we show that in general the problem remains NP-hard both on temporal split and temporal permutation graphs, but we also spot promising islands of fixed-parameter tractability particularly based on parameterizations that measure the amount of "change over time".
\end{abstract}

Keywords: Temporal graphs, Connectivity problems, Special graph classes, NP-hardness, Fixed-parameter tractability.

\section{Introduction}

Finding a smallest set of vertices whose deletion disconnects two designated vertices - the separation problem - is a fundamental problem in algorithmic graph theory. The problem, which is a backbone of numerous applications related to network connectivity, is well-known to be polynomial-time solvable in (static) graphs. Driven by the need of understanding and mastering dynamically changing network structures, in recent years the study of temporal graphs - graphs with a fixed vertex set but edge sets that may change over discrete time steps - has enjoyed an enormous growth. One of the earliest systematic studies on temporal graphs dealt with the separation problem [18], where it turned out to be NP-hard. This motivates the study of parameterized complexity aspects as well as of the complexity behavior on special temporal graph

\footnotetext{
${ }^{*}$ H. Molter was supported by the DFG, project MATE (NI 369/17), and by the ISF, grant No. 1070/20. Main part of this work was done while H. Molter was affiliated with TU Berlin. M. Renken was supported by the DFG, project MATE (NI 369/17).
} 
classes $[13,23]$. Continuing and extending this line of research, we provide a first in-depth study on temporal versions of split and permutation graphs, two classes of perfect graphs on which many generally NP-hard problems become polynomial-time solvable [5, 15]. We present both intractability as well as (fixed-parameter) tractability results.

Formally, a temporal graph is an ordered triple $\mathcal{G}=(V, \mathcal{E}, \tau)$, where $V$ denotes the set of vertices, $\mathcal{E} \subseteq\left(\begin{array}{c}V \\ 2\end{array}\right) \times\{1,2, \ldots, \tau\}$ the set of time-edges where $(\{v, w\}, t) \in \mathcal{E}$ represents an edge between vertices $v$ and $w$ available at time $t$, and $\tau \in \mathbb{N}$ is the maximum time label. We can think of it as a series of $\tau$ static graphs, called layers. The graph containing the union of the edges of all layers is called the underlying graph of $\mathcal{G}$.

Recently, connectivity and path-related problems have been extensively studied on temporal graphs $[2,6,7,9,10,11,13,20,23]$. In the temporal setting, paths, walks, and reachability are defined in a time-respecting way [18]: A temporal $(s, z)$-walk (or temporal walk) of length $k$ from vertex $s=v_{0}$ to vertex $z=v_{k}$ in a temporal graph $\mathcal{G}$ is a sequence $P=\left(\left(v_{i-1}, v_{i}, t_{i}\right)\right)_{i=1}^{k}$ such that $\left(\left\{v_{i-1}, v_{i}\right\}, t_{i}\right)$ is a time-edge of $\mathcal{G}$ for all $i \in\{1,2, \ldots, k\}$ and $t_{i} \leq t_{i+1}$ for all $i \in$ $\{1,2, \ldots, k-1\} .{ }^{1}$ A temporal walk is a temporal path if it visits every vertex at most once.

We study the Temporal $(s, z)$-Separation problem. Here, a temporal $(s, z)$-separator is a set of vertices (not containing $s$ and $z$ ) whose removal destroys all temporal paths from $s$ to $z$.

\section{TEMPoRAL $(s, z)$-SEPARATION}

Input: $\quad$ A temporal graph $\mathcal{G}=(V, \mathcal{E}, \tau)$, two distinct vertices $s, z \in V$, and $k \in \mathbb{N}$.

Question: Does $\mathcal{G}$ admit a temporal $(s, z)$-separator of size at most $k$ ?

Temporal $(s, z)$-Separation is NP-complete [18] and W[1]-hard when parameterized by the separator size $k$ [23]. On the positive side, one can verify a solution in $\mathcal{O}(|\mathcal{G}|)$ time (see e.g. Bui-Xuan et al. [6]). Zschoche et al. [23] investigated the differences between the computational complexity of finding temporal separators that remove (non-strict) temporal paths vs. strict temporal paths. Fluschnik et al. [13] studied the impact of restrictions on the layers or the underlying graph on the computational complexity of TEMPORAL $(s, z)$-SEPARATION and found that even under severe restrictions Temporal $(s, z)$-SEPARATION remains NP-complete. In particular, the problems stays NP-complete and W[1]-hard when parameterized by the separator size even if every layer contains only one edge and for several restrictions of the underlying graph [13]. They further investigated the case where every layer of the temporal graph is a unit interval graph and obtained fixed-parameter tractability for TEMPORAL $(s, z)$-SEPARATion when parameterized by the lifetime $\tau$ and the so-called "shuffle number", a parameter that measures how much the relative order of the intervals changes over time. This result initiated research on the amount of "change over time" measured by a parameter that is tailored to the graph class into which all layers fall.

In our work, we follow this paradigm of restricting each layer to a certain graph class and measuring the amount of change over time with parameters that are tailored to the graph class of the layers. More specifically, we investigate the complexity of TEMPORAL $(s, z)$-SEPARATion on temporal graphs where every layer is a split graph or every layer is a permutation graph.

Temporal split graphs. In a split graph, the vertex set can be partitioned into a clique and an independent set. Split graphs can be used to model an idealized form of core-periphery structures, in which there exists a densely connected core and a periphery that only has connections to that core [4]. Core-periphery structures can be observed in social contact networks in which one group of people meets at some location, forming a fully connected core. Meanwhile, other people associated with the group may have contact with some of its members, but

\footnotetext{
${ }^{1}$ Such walks are also called "non-strict", whereas "strict" walks require $t_{i}<t_{i+1}$. We focus on non-strict walks in this work.
} 
otherwise do not have any interactions relevant to the observed network. Such applications are naturally subject to change over time, for example due to vertices entering or leaving the network. ${ }^{2}$ This temporal aspect is captured by temporal split graphs, where each layer has a separate core-periphery split.

We prove that TEMPORAL $(s, z)$-SEPARATION remains NP-complete on temporal split graphs, even with only four layers. On the positive side, we show that TEMPORAL $(s, z)$-SEPARATion is solvable in polynomial time on temporal graphs where the partition of the vertices into a clique and an independent set stays the same in every layer. We use this as a basis for a "distanceto-triviality"-parameterization [16, 21], also motivated by the assumption that in application cases the core-periphery structure of a network will roughly stay intact over time with only few changes. Intuitively, we parameterize on how many vertices may switch between the two parts over time and use this parameterization to obtain fixed-parameter tractability results. Formally, we show fixed-parameter tractability for the combined parameter "number of vertices different from $s$ and $z$ that switch between the clique and the independent set at some point in time" and the lifetime $\tau$.

Temporal permutation graphs. A permutation graph on an ordered set of vertices is defined by a permutation of that ordering. Two vertices are connected by an edge if their relative order is inverted by the permutation. They were introduced by Even et al. [12] and appear in integrated circuit design [22], memory layout optimization [12], and other applications [15]. In a temporal permutation graph, the edges of each layer are given by a separate permutation. We prove that TEMPoral $(s, z)$-SePARATION remains NP-complete on temporal permutation graphs. We then parameterize on how much the permutation changes over time to obtain fixedparameter tractability results. We use the Kendall tau distance [19] to measure the dissimilarity of the permutations. The Kendall tau distance is a metric that counts the number of pairwise disagreements between two total orderings; it is also known as "bubble sort distance". More precisely, we obtain fixed-parameter tractability for the combined parameter "sum of Kendall tau distances between consecutive permutations" and the separator size $k$. We remark that in a similar context, the Kendall tau distance has also been used by Fluschnik et al. [13] to measure the amount of change over time in a temporal graph.

\section{Split Graphs}

In this section, we study the computational complexity of finding temporal separators in temporal split graphs. Split graphs represent an idealized model of core-periphery structures with a well-connected core and a periphery only connected to that core [4]. They also constitute the majority of all chordal graphs [1].

Formally, a graph $G=(V, E)$ is called a split graph if $V$ can be partitioned into two sets $C, I$ such that $C$ induces a clique and $I$ induces an independent set. Then, $(C, I)$ is called a split partition of $G$. In general, a split graph may admit multiple split partitions. A temporal split graph is a temporal graph $\mathcal{G}$ of which every layer is a split graph. A temporal split partition $\left(C_{t}, I_{t}\right)_{t=1}^{\tau}$ then contains a split partition of every layer of $\mathcal{G}$.

\subsection{Hardness Results}

The fact that TEMPorAL $(s, z)$-SEPARATION on temporal split graphs is NP-hard can be derived from a result of Fluschnik et al. [13] stating that TEMPORAL $(s, z)$-SEPARATion is hard on

\footnotetext{
${ }^{2}$ While the vertex set of a temporal graph formally remains unchanged, isolated vertices are equivalent to non-existing vertices as far as separators are concerned.
} 


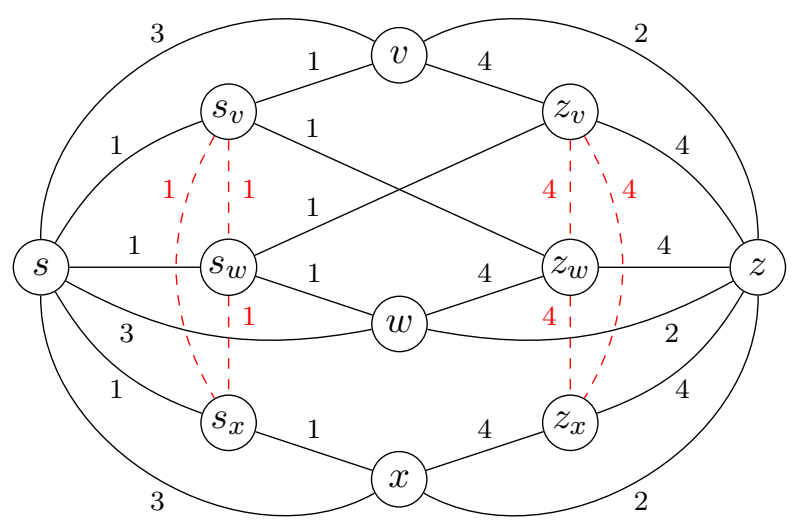

Figure 1: The graph $\mathcal{G}^{\prime \prime}$ for $G=(\{v, w, x\},\{\{v, w\}\})$ in Theorem 2.1. The edges from $\mathcal{E}_{\alpha}$ are in solid black, and the edges from $\mathcal{E}_{C}$ are in red and dashed.

temporal graphs containing a single edge per layer. This is due to the fact that a graph with a single edge is clearly a split graph.

We now strengthen this result, showing that TEMPoral $(s, z)$-SEPARATion is NP-hard on temporal split graphs with only a constant number of layers. We do this by building on a reduction by Zschoche et al. [23] showing NP-hardness of Temporal $(s, z)$-SEPARATion on general temporal graphs.

Here and in the following we use "separator" as a shorthand for "temporal $(s, z)$-separator" when no ambiguity arises.

Theorem 2.1. Temporal $(s, z)$-Separation is NP-hard on temporal split graphs with four layers.

Proof. We prove this by building on the proof by Zschoche et al. [23] showing NP-hardness of TEMPoral $(s, z)$-SEPARATiOn on general temporal graphs. Their proof employs a polynomialtime reduction from VERTEX COVER. We extend this reduction so that it produces a TEMPORAL $(s, z)$-SEPARATION instance on a temporal split graph with $\tau=4$.

Let $\mathcal{I}:=(G=(V, E), k)$ be a Vertex Cover instance and $n:=|V|$. Construct a Tempo$\operatorname{RAL}(s, z)$-Separation instance $\mathcal{I}^{\prime}:=\left(\mathcal{G}^{\prime}=\left(V^{\prime}, \mathcal{E}^{\prime}, 2\right), s, z, n+k\right)$, where $V^{\prime}=V \cup\left\{s_{v}, z_{v} \mid v \in\right.$ $V\} \cup\{s, z\}$ are the vertices and the time-edges are defined as

$$
\begin{aligned}
\mathcal{E}^{\prime}:= & \left\{\left(\left\{s, s_{v}\right\}, 1\right),\left(\left\{s_{v}, v\right\}, 1\right),\left(\left\{v, z_{v}\right\}, 2\right),\left(\left\{z_{v}, z\right\}, 2\right),(\{s, v\}, 2),(\{v, z\}, 1) \mid v \in V\right\} \cup \\
& \left\{\left(\left\{s_{v}, z_{w}\right\}, 1\right),\left(\left\{s_{w}, z_{v}\right\}, 1\right) \mid\{v, w\} \in E\right\} .
\end{aligned}
$$

Note that Zschoche et al. [23] showed that deciding $\mathcal{I}^{\prime}$ is NP-complete.

The idea is now to divide each of the layers of $\mathcal{G}^{\prime}$ into two layers in a way that turns all layers into split graphs while not changing the set of temporal $(s, z)$-separators. More specifically, we construct another Temporal $(s, z)$-Separation instance $\mathcal{I}^{\prime \prime}:=\left(\mathcal{G}^{\prime \prime}=\left(V^{\prime}, \mathcal{E}^{\prime \prime}, 4\right), s, z, n+k\right)$, where the time-edges are defined as

$$
\begin{aligned}
\mathcal{E}^{\prime \prime}:= & \mathcal{E}_{\alpha} \cup \mathcal{E}_{C}, \text { where } \\
\mathcal{E}_{\alpha}:= & \left\{\left(\left\{s, s_{v}\right\}, 1\right),\left(\left\{s_{v}, v\right\}, 1\right),\left(\left\{v, z_{v}\right\}, 4\right),\left(\left\{z_{v}, z\right\}, 4\right),(\{s, v\}, 3),(\{v, z\}, 2) \mid v \in V\right\} \cup \\
& \left\{\left(\left\{s_{v}, z_{w}\right\}, 1\right),\left(\left\{s_{w}, z_{v}\right\}, 1\right) \mid\{v, w\} \in E\right\}, \\
\mathcal{E}_{C}:= & \left\{\left(\left\{s_{v}, s_{w}\right\}, 1\right),\left(\left\{z_{v}, z_{w}\right\}, 4\right) \mid v, w \in V, v \neq w\right\} .
\end{aligned}
$$


The time-edges in $\mathcal{E}_{\alpha}$ are the same edges as in $\mathcal{E}^{\prime}$, except that they are distributed over four layers. The time-edges in $\mathcal{E}_{C}$ induce cliques in layers 1 and 4 , turning them into split graphs. The constructed temporal graph is visualized in Fig. 1.

Note that $\left|\mathcal{E}^{\prime \prime}\right|=\left|\mathcal{E}^{\prime}\right|+2\left(\begin{array}{c}n \\ 2\end{array}\right)$ and $\mathcal{I}^{\prime \prime}$ can be computed in polynomial time. All four layers of $\mathcal{G}^{\prime \prime}$ are split graphs: the time-edges in $\mathcal{E}_{C}$ exactly form a clique each in layers 1 and 4 ; in layer $2,\{z\}$ is the clique, and in layer $3,\{s\}$ is the clique. The time-edges in $\mathcal{E}_{\alpha}$ all have a vertex from their layer's clique as one endpoint, thus not connecting any two vertices outside of it.

Correctness. We prove that $\mathcal{I}^{\prime}$ is a yes-instance if and only if $\mathcal{I}^{\prime \prime}$ is a yes-instance by showing that a vertex set is a $(s, z)$-separator of $\mathcal{G}^{\prime}$ if and only if it is also a $(s, z)$-separator of $\mathcal{G}^{\prime \prime}$.

First, we note that the temporal $(s, z)$-paths in $\mathcal{E}^{\prime}$ and $\mathcal{E}_{\alpha}$ are identical except for their time labels: every time-edge in layer 1 of $\mathcal{G}^{\prime}$ has a corresponding time-edge from $\mathcal{E}_{\alpha}$ between the same vertices in layer 1 or 2 of $\mathcal{G}^{\prime \prime}$, and in layer 2 of $\mathcal{G}^{\prime}$, each has a corresponding time-edge from $\mathcal{E}_{\alpha}$ in layer 3 or 4 of $\mathcal{G}^{\prime \prime}$. All time-edges in $\mathcal{E}_{\alpha}$ correspond to one in $\mathcal{E}^{\prime}$ in this way. This means that every temporal path in $\mathcal{E}_{\alpha}$ has a corresponding path in $\mathcal{E}^{\prime}$ that also has ascending time labels. Conversely, a temporal path in $\mathcal{E}^{\prime}$ will have a corresponding path in $\mathcal{E}_{\alpha}$ that first only passes through time-edges with labels 1 or 2 , and then through time-edges with labels 3 or 4 . Also, all time-edges with label 2 in $\mathcal{E}_{\alpha}$ are connected to $z$, and all time-edges with label 3 in $\mathcal{E}_{\alpha}$ are connected to $s$. So an $(s, z)$-path cannot pass any edges after an edge in layer 2 or before an edge in layer 3 , making impossible any $(s, z)$-path with an edge from layer 2 before one from layer 1 , or an edge from layer 4 before one from layer 3 . Hence, all paths in $\mathcal{E}_{\alpha}$ corresponding to a temporal $(s, z)$-path in $\mathcal{E}^{\prime}$ are also valid temporal $(s, z)$-paths.

$\Rightarrow$ : We show that the addition of $\mathcal{E}_{C}$ does not result in any new temporal $(s, z)$-paths that can not be shortened to one already existing in $\mathcal{E}_{\alpha}$, and by extension, in $\mathcal{E}^{\prime}$. In layer 1 , there is a time-edge from $s$ to every vertex that is part of the clique. Any path that takes one or more of the time-edges in layer 1 of $\mathcal{E}_{C}$ can therefore be shortened to a path that goes directly from $s$ to the last vertex reached by taking one of the new time-edges, eliminating all of these new edges from the path. Equivalently, all vertices in the clique in layer 4 have a time-edge to $z$. So instead of taking any of the time-edges leading to other members of the clique, any $(s, z)$-path could just directly take the edge to $z$. This means that there are no temporal $(s, z)$-paths in $\mathcal{G}^{\prime \prime}$ that do not get cut by an $(s, z)$-separator of $\mathcal{G}^{\prime}$.

$\Leftarrow$ : Any $(s, z)$-separator of $\mathcal{G}^{\prime \prime}$ must cut all temporal $(s, z)$-paths in $\mathcal{E}_{\alpha}$, thereby also cutting all temporal $(s, z)$-paths in $\mathcal{E}^{\prime}$, making it a $(s, z)$-separator of $\mathcal{G}^{\prime}$, too.

Theorem 2.1 shows that TEMPORAL $(s, z)$-SEPARATION on temporal split graphs is NP-hard for $\tau \geq 4$. Clearly, Temporal $(s, z)$-Separation is polynomial-time solvable for $\tau=1$. The computational complexity for the cases $\tau=2$ and $\tau=3$ remains open.

\section{$2.2 \quad$ Fixed-Parameter Tractability Results}

The defining characteristic of temporal split graphs is that for each layer $t$ they can be split into a clique $C_{t}$ and an independent set $I_{t}$. While temporal split graphs allow for changes of this partition, for several application scenarios described in the introduction it is reasonable to assume that only a few of these changes occur while most vertices retain their role throughout all layers.

We will prove that Temporal $(s, z)$-Separation can be solved efficiently in this setting, that is, if the number of switching vertices $\bigcup_{t, t^{\prime}} C_{t} \cap I_{t^{\prime}}$ is low. To this end, we first prove that Temporal $(s, z)$-SePARATion is polynomial-time solvable if the only switching vertices are $s$ and $z$. 
Lemma 2.2. Let $\mathcal{G}=(V \cup\{s, z\}, \mathcal{E}, \tau)$ be a temporal split graph with a given temporal split partition having no switching vertices except possibly $s$ and $z$. Then all minimal temporal $(s, z)-$ separators in $\mathcal{G}$ can be found in $\mathcal{O}(|\mathcal{G}| \cdot \tau)$ time.

Proof. We assume that there is never an edge between $s$ and $z$, otherwise the problem is trivial. Let the given temporal split partition be $\left(C_{t}, I_{t}\right)_{t}$. Let $C:=C_{t} \backslash\{s, z\}$ and $I:=I_{t} \backslash\{s, z\}$ (for some and thus all layers $t)$. We show that all minimal temporal $(s, z)$-separators are given by the set

$$
\begin{aligned}
& \mathcal{S}:=\left\{\bigcup_{0<t \leq i}\left(N_{G_{t}}(s) \cap C\right) \cup \bigcup_{i<t \leq \tau}\left(N_{G_{t}}(z) \cap C\right) \cup T \mid 0 \leq i \leq \tau\right\}, \text { where } \\
& T:=\bigcup_{1 \leq t \leq t^{\prime} \leq \tau} N_{G_{t}}(s) \cap N_{G_{t^{\prime}}}(z) .
\end{aligned}
$$

The set $\mathcal{S}$ can be constructed in $\mathcal{O}(|\mathcal{G}| \cdot \tau)$ time and contains at most $\tau+1$ elements. This proves the stated time bound. It remains to verify that $\mathcal{S}$ contains all minimal separators.

First, note that $T$ contains exactly those vertices which form temporal $(s, z)$-paths of length 2. Thus $T$ has to be contained in any separator.

So it only remains to consider temporal $(s, z)$-paths of length at least 3 . If such a path $P$ contains a vertex $v \in I$, then let $(\{u, v\}, t)$ and $\left(\{v, w\}, t^{\prime}\right)$ be the two time-edges of $P$ containing $v$. Then we must have $u \in C$ or $w \in C$ and thus the above two time-edges can be replaced by either $(\{u, w\}, t)$ or $\left(\{u, w\}, t^{\prime}\right)$, shortening $P$ by one. Consequently, if $\mathcal{G}$ contains a temporal $(s, z)$-path of length at least 3 , then there is also a temporal $(s, z)$-path in $\mathcal{G}-I .{ }^{3}$ So it suffices to consider temporal paths in $\mathcal{G}-I$.

Thus it becomes clear that each element of $\mathcal{S}$ is in fact a temporal $(s, z)$-separator. Now let $S$ be an arbitrary temporal $(s, z)$-separator and $i$ maximal with $S \supseteq \bigcup_{0<t \leq i} N_{G_{t}}(s) \cap C$. Thus, $S$ does not contain some vertex $v \in N_{G_{i+1}}(s) \cap C$. Then, $\mathcal{G}-S$ contains a path from $s$ to every vertex in $C \backslash S$ since $v$ is connected to all other vertices in $C$. So starting from layer $i+1$, all edges to $z$ from a vertex in $C \backslash S$ would complete a temporal path from $s$ to $z$. Hence, $S \supseteq \bigcup_{i<t \leq \tau} N_{G_{t}}(z) \cap C$, concluding the proof.

Next, we first show that this is still an NP-hard problem and then give an FPT-algorithm for the solution size parameter.

Proposition 2.3. For temporal split graphs, it is NP-hard to compute a minimum-size set of switching vertices.

Proof. We present a polynomial-time reduction from Vertex Cover [14]. Let $(H=(U, F), h)$ be an instance of VERTEX COVER, then we construct a temporal split graph $\mathcal{G}=(V, \mathcal{E}, \tau)$ as follows. We set $V:=\left\{u_{1}, u_{2} \mid u \in U\right\}$. Now we create $|F|+1$ layers. In the first layer, we create a clique on the vertices $\left\{u_{1} \mid u \in U\right\}$ and connect $u_{1}$ with $u_{2}$ for all $u \in U$. Formally,

$$
E_{1}(\mathcal{G}):=\left\{\left\{u_{1}, v_{1}\right\} \mid u, v \in U \wedge u \neq v\right\} \cup\left\{\left\{u_{1}, u_{2}\right\} \mid u \in U\right\} .
$$

Now let the edges of $H$ be ordered in some fixed but arbitrary way, that is, $F=\left\{e_{1}, e_{2}, \ldots, e_{|F|}\right\}$. For $i \in[|F|]$, we create layer $i+1$ with the following edge set:

$$
E_{i+1}(\mathcal{G}):=\left\{\left\{u_{1}, v_{1}\right\} \mid u, v \in U \wedge u \neq v \wedge\{u, v\} \neq e_{i}\right\} .
$$

This graph can clearly be constructed in polynomial time and it is easy to check that it is in fact a temporal split graph.

\footnotetext{
${ }^{3}$ We denote by $\mathcal{G}-X$ the temporal graph resulting from removing vertices in $X$ from the vertex set of $\mathcal{G}$.
} 
Correctness. In the following we show that the cardinality of a minimum-size set of switching vertices for $\mathcal{G}$ is at most $h$ if and only if $H$ admits a vertex cover of size at most $h$.

We start with some observations about the constructed temporal split graph $\mathcal{G}$. In the first layer, we have a unique valid partition of the vertices into a clique and an independent set: $C_{1}=\left\{u_{1} \mid u \in U\right\}$ forms the clique and $I_{1}=\left\{u_{2} \mid u \in U\right\}$ forms the independent set. Vertices in $I_{1}$ may stay in the independent set in all other layers as well, however in every layer exactly one pair of vertices in $C_{1}$ misses the connecting edge, hence at least one of them needs to be in the independent set of that layer. Intuitively, these vertices which need to switch correspond to a vertex cover in $H$.

$\Rightarrow$ : Assume $H$ contains a vertex cover $X \subset U$ of size at most $h$. Then we claim that the set $Y=\left\{u_{1} \mid u \in X\right\}$ forms a set of switching vertices of size $h$ for $\mathcal{G}$. The following partitions of the vertices into a clique and an independent set for all layer witness this. As already described earlier, we have that $C_{1}=\left\{u_{1} \mid u \in U\right\}$ and $I_{1}=\left\{u_{2} \mid u \in U\right\}$. For layer $i$ with $i>1$ we set $C_{i}=C_{1} \backslash\left\{u_{1} \mid u \in X \cap e_{i-1}\right\}$ and $I_{i}=I_{1} \cup\left\{u_{1} \mid u \in X \cap e_{i-1}\right\}$. First, we can observe that the vertices in $Y$ are the only switching vertices. Furthermore, since $X$ is a vertex cover in $H$ we have that for each layer $i$, at least one of the two vertices from $C_{1}$ that are not connected by an edge is in $I_{i}$. Otherwise the edge $e_{i-1}$ would not be covered by $X$.

$\Leftarrow$ : Assume we find a set of switching vertices $Y$ of size at most $h$ for $\mathcal{G}$. First, observe that $Y \cap I_{1}=\emptyset$ since in none of the layers, a vertex from $I_{1}$ can be in the clique. We claim that $X=\left\{u \mid u_{1} \in Y\right\}$ is a vertex cover for $H$. Assume for contradiction that there is an edge $e_{i} \in F$ such that $e_{i} \cap X=\emptyset$. Let $e_{i}=\{u, v\}$. Then we have that in layer $i+1$ vertices $u_{1}, v_{1}$ are both in the clique, since they are in $C_{1}$ and not switching. However, by construction there is no edge between $u_{1}$ and $v_{1}$ in layer $i+1$. Hence, not both vertices can be part of the clique in this layer. A contradiction.

Proposition 2.4. For a temporal split graph $\mathcal{G}=(V, \mathcal{E}, \tau)$, one can find a temporal split partition minimizing the number of switching vertices in $\mathcal{O}\left(|\mathcal{E}|+\tau \cdot|V|+|V|^{2} \cdot\left(1.2738^{p}+p \cdot|V|\right)\right)$ time, where $p$ is the minimum number of switching vertices.

Proof. Let $G_{t}$ be any layer of $\mathcal{G}$. By a result of Heggernes and Mancini [17], we can find in $\mathcal{O}\left(\left|G_{t}\right|\right)$ time a partition $V=\hat{C}_{t} \cup \hat{I}_{t} \cup Q_{t}$ (each part possibly empty) such that $\hat{C}_{t} \subseteq C$ and $\hat{I}_{t} \subseteq I$ for every split partition $(C, I)$ of $G_{t}$. Furthermore, $Q_{t}$ forms either a clique or an independent set and every vertex of $Q_{t}$ is adjacent to all vertices of $\hat{C}_{t}$ and to no vertex of $\hat{I}_{t}$. If $Q_{t}$ is a clique, then all possible split partitions of $G_{t}$ are clearly given by

$$
\left\{(V \backslash I, I)\left|\hat{I}_{t} \subseteq I \subseteq \hat{I}_{t} \cup Q_{t},\right| I \cap Q_{t} \mid \leq 1\right\}
$$

and if $Q_{t}$ is an independent set, then all split partitions of $G_{t}$ are given by

$$
\left\{(C, V \backslash C)\left|\hat{C}_{t} \subseteq C \subseteq \hat{C}_{t} \cup Q_{t},\right| C \cap Q_{t} \mid \leq 1\right\} .
$$

Suppose now we are given a temporal split partition $\left(C_{t}, I_{t}\right)$ of $\mathcal{G}$. Denote by $T$ the set of switching vertices of this partition, and by $C:=\bigcap_{t} C_{t}$ and $I:=\bigcap_{t} I_{t}$ the set of non-switching vertices.

Let $Q_{\cap}:=\bigcap_{t=1}^{\tau} Q_{t}$. If there exist $t, t^{\prime}$ such that $Q_{\cap}$ is a clique in $G_{t}$ but an independent set in $G_{t^{\prime}}$, then all but two vertices in $Q_{\cap}$ must be switching vertices, as $Q_{\cap} \cap I_{t}$ and $Q_{\cap} \cap C_{t^{\prime}}$ can each contain at most one vertex.

Clearly, all vertices in $S:=\bigcup_{t \neq t^{\prime}} \hat{C}_{t} \cap \hat{I}_{t^{\prime}}$ must also be switching vertices. The remaining vertices $\tilde{V}:=V \backslash\left(Q_{\cap} \cup S\right)$ can be partitioned into two sets. The first one, $\tilde{C}:=\bigcap_{t}\left(\hat{C}_{t} \cup Q_{t}\right) \backslash Q_{\cap}$ contains vertices which are sometimes in $\hat{C}_{t}$ and sometimes in $Q_{t}$. The second one, $\tilde{I}:=$ $\bigcap_{t}\left(\hat{I}_{t} \cup Q_{t}\right) \backslash Q_{\cap}$ contains vertices which are sometimes in $\hat{I}_{t}$ and sometimes in $Q_{t}$. 
We build an auxiliary graph $A_{C}$ as follows: Start with the vertex set $\tilde{C}$. Additionally, if there is a vertex $q_{C} \in Q_{\cap} \cap C$, then also add $q_{C}$ to the vertices of $A_{C}$. Then connect any two vertices $v, w$ in $A_{C}$ if and only if they are not connected in some layer of $\mathcal{G}$. Analogously, we build another graph $A_{I}$. The vertex set of $A_{I}$ is given by $\tilde{I}$ and, possibly, the unique vertex $q_{I} \in Q_{\cap} \cap I$. We connect two vertices $v, w$ in $A_{I}$ if and only if they are connected in some layer of $\mathcal{G}$.

Observe that $T \cap V\left(A_{C}\right)$ forms a vertex cover of $A_{C}$ : if $\{v, w\} \in E\left(A_{C}\right)$, then there is a layer with $\{v, w\} \notin E\left(G_{t}\right)$, thus at least one of $v, w$ must be in $I_{t}$ and thus in $T$, since every vertex of $A_{C}$ is contained in some $C_{t^{\prime}}$. Analogously, $T \cap V\left(A_{I}\right)$ forms a vertex cover of $A_{I}$ : if $\{v, w\} \in E\left(A_{I}\right)$, then there is a layer with $\{v, w\} \in E\left(G_{t}\right)$, therefore one of $v, w$ is in $C_{t} \cap \tilde{I} \subseteq T$. Obviously, these vertex covers do not contain $q_{C}$ or $q_{I}$.

We claim that we can use the converse direction to obtain a temporal split partition of $\mathcal{G}$. First, we compute $\hat{C}_{t}, \hat{I}_{t}$, and $Q_{t}$ for each layer $G_{t}$. Then we select up to two vertices $q_{C}, q_{I} \in Q_{\cap}$. Having these, we build the two auxiliary graphs $A_{C}$ and $A_{I}$ as above. We then compute two vertex covers $T_{C}$ and $T_{I}$, excluding $q_{C}$ and $q_{I}$ if they exist. The temporal split partition $\left(C_{t}, I_{t}\right)_{t}$ is then constructed as follows. Clearly, $C_{t} \supseteq \hat{C}_{t}$ and $I_{t} \supseteq \hat{I}_{t}$. If $Q_{t}=\emptyset$, then this already defines $\left(C_{t}, I_{t}\right)$.

If $Q_{t}$ is a clique in $G_{t}$, then we add to $I_{t}$ any vertices in $V\left(A_{I}\right) \backslash T_{I}$ and all remaining vertices to $C_{t}$. Since $T_{I}$ is a vertex cover of $A_{I}, Q_{t} \cap\left(V\left(A_{I}\right) \backslash T_{I}\right)$ contains at most one vertex, making this a valid split partition of $G_{t}$.If $Q_{t}$ is an independent set in $G_{t}$, then we analogously add to $C_{t}$ any vertices in $V\left(A_{C}\right) \backslash T_{C}$ and all remaining vertices to $I_{t}$.

It is not difficult to check that the switching vertices of the resulting temporal split partition are exactly the vertices in $S \cup T_{C} \cup T_{I} \cup\left(Q_{\cap} \backslash\left\{q_{C}, q_{I}\right\}\right)$. To ensure that the resulting temporal split partition has a minimum number of switching vertices, we can simply try all possible choices for $q_{C}$ and $q_{I}$, each time computing minimum vertex covers $T_{I}$ and $T_{C}$.

The required time to compute all $\hat{C}_{t}, \hat{I}_{t}, Q_{t}$ as well as $Q_{\cap}, S, A_{C}$ and $A_{I}$ is in $\mathcal{O}\left(\sum_{t}\left|G_{t}\right|\right) \subseteq$ $\mathcal{O}(|\mathcal{E}|+\tau|V|)$. For each of the $\mathcal{O}\left(|V|^{2}\right)$ possible ways of choosing $q_{C}, q_{I}$, we need to compute minimum size vertex covers for $A_{C}$ and $A_{I}$. Each of these computations is possible in $\mathcal{O}\left(1.2738^{p}+p \cdot|V|\right)$ time [8]. The overall time requirement is thus in $\mathcal{O}\left(|\mathcal{E}|+\tau \cdot|V|+|V|^{2}\right.$. $\left.\left(1.2738^{p}+p \cdot|V|\right)\right)$.

Based on Lemma 2.2 and Proposition 2.4, we can now show a fixed-parameter algorithm for the parameter lifetime $\tau$ combined with the parameter number $p$ of switching vertices apart from $s, z$.

Theorem 2.5. Let $\mathcal{G}$ be a temporal split graph with at most $p$ switching vertices apart from $s$ and $z$. Then Temporal $(s, z)$-Separation on $\mathcal{G}$ can be solved in $\mathcal{O}\left((\tau+1)^{3^{p}(p+1)}|\mathcal{G}|+1.2738^{p}\right.$. $\left.|V|^{2}+p \cdot|V|^{3}\right)$ time.

Proof. We begin with using Proposition 2.4 to compute a temporal split partition of $\mathcal{G}$. We then use induction to show that for all values of $p$ there are at most $(\tau+1)^{3^{p}(p+1)}$ minimal temporal $(s, z)$-separators, which can all be found in $D(\tau+1)^{3^{p}(p+1)}|\mathcal{G}|$ time for some constant $D$.

For the case $p=0$, by Lemma 2.2, we can find all minimal separators of which there are at most $\tau+1$, in $\mathcal{O}(|\mathcal{G}| \cdot \tau)$ time.

Now for the induction step suppose that our claim holds whenever the number of switching vertices (apart from $s, z)$ is at most $p-1$. We choose a switching vertex $v$ from $\mathcal{G}(v \notin\{s, z\})$. The subgraph $\mathcal{G}-v$ then contains $p-1$ switching vertices apart from $s, z$, therefore we can find all its minimal separators in $D(\tau+1)^{3^{p-1} p}|\mathcal{G}|$ time. Since a separator of $\mathcal{G}$ is also a separator of $\mathcal{G}-v$, every minimal separator of $\mathcal{G}$ must contain a separator of $\mathcal{G}-v$. We will base our separators for $\mathcal{G}$ on the minimal separators of $\mathcal{G}-v$, henceforth called base separators, by finding 
all possible combinations of vertices that can be added to turn them into minimal separators of $\mathcal{G}$.

Because we only added $v$, all temporal $(s, z)$-paths left in $\mathcal{G}$ after removing a separator of $\mathcal{G}-v$ must pass through $v$. Thus any separator of $\mathcal{G}$ must either contain $v$ or some other set of vertices that cuts all these paths. To do the latter, it has to ensure that all remaining temporal $(s, v)$ paths arrive after the latest layer in which a temporal $(v, z)$-path can begin. In other words, such a separator of $\mathcal{G}$ needs to contain a temporal $(s, v)$-separator for the layers from 1 to some layer $t$, and a temporal $(v, z)$-separator from $t+1$ to $\tau$. We can compute all minimal separators of this form by applying the induction hypothesis to enumerate all temporal $(s, v)$-separators in layers 1 through $t$ of $\mathcal{G}-\{z\}$ and all temporal $(v, z)$-separators in layers $t+1$ through $\tau$ of $\mathcal{G}-\{s\}$. Note that both of these are temporal split graphs with at most $p-1$ switching vertices (not counting $s, v$, and $z$ ).

So for any given $t$, there are no more than $\left((\tau+1)^{3^{p-1} p}\right)^{2}$ possible separator combinations. Additionally we have the option of simply taking $v$. As there are $\tau+1$ options for $t$ and $(\tau+$ $1)^{3^{p-1} p}$ base separators to choose from, the overall number of minimal temporal $(s, z)$-separators is thus upper-bounded by

$$
(\tau+1)^{3^{p-1} p}\left(\left((\tau+1)^{3^{p-1} p}\right)^{2}(\tau+1)+1\right) \leq(\tau+1)^{3^{p}(p+1)} .
$$

We require $D(\tau+1)^{3^{p-1}} p|\mathcal{G}|$ time to find all base separators. In addition, for each $t \in\{0, \ldots, \tau\}$ we need $2 D(\tau+1)^{3^{p-1} p}|\mathcal{G}|$ time to compute all minimal $(s, v)$ - and $(v, z)$-separators. Afterwards we need $\mathcal{O}(|V|)$ time to output each found separator. The overall time required thus is

$$
\begin{aligned}
& D(\tau+1)^{3^{p-1} p}|\mathcal{G}| \cdot(1+2(\tau+1))+D(\tau+1)^{3^{p-1} 2 p}|V| \\
\leq & D(\tau+1)^{3^{p}(p+1)}|\mathcal{G}|
\end{aligned}
$$

where we assumed $\tau \geq 3$. This completes the induction. Together with the time required for Proposition 2.4, we obtain an overall upper time bound of

$$
\begin{aligned}
& \mathcal{O}\left((\tau+1)^{3^{p}(p+1)}|\mathcal{G}|+|\mathcal{E}|+\tau \cdot|V|+|V|^{2} \cdot\left(1.2738^{p}+p \cdot|V|\right)\right) \\
\subseteq & \mathcal{O}\left((\tau+1)^{3^{p}(p+1)}|\mathcal{G}|+1.2738^{p} \cdot|V|^{2}+p \cdot|V|^{3}\right) .
\end{aligned}
$$

We leave open whether for the single parameter number $p$ of switching vertices TEMPORAL $(s, z)$-SEPARATION is fixed-parameter tractable.

\section{Permutation Graphs}

In this section, we investigate the complexity of finding temporal separators in temporal permutation graphs. A permutation graph is defined by a ordered set of vertices (say $1, \ldots, n$ ) and any permutation of that vertex set. Two vertices are connected by an edge if and only if their relative order is inverted by the permutation.

Formally, we call a graph $G=(V, E)$ with vertex set $V=[n]:=\{1,2, \ldots, n\}$ a permutation graph, if there exists a permutation $\pi: V \rightarrow V$ of the vertices such that for any two vertices $v<w$, we have $\{v, w\} \in E$ if and only if $\pi(w)<\pi(v)$. Clearly, $\pi$ is then uniquely determined by $G$. Note that we follow the definition of Even et al. [12] in which the vertices are already labeled; some authors also define a permutation graph as any (unlabeled) graph for which such a labeling can be found [15]. 


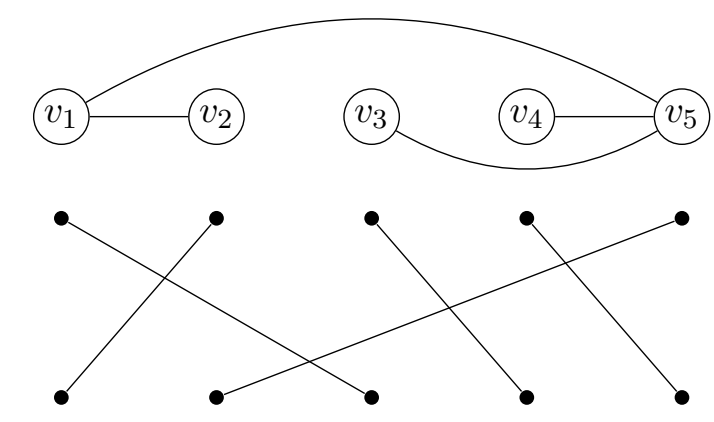

Figure 2: An example of a permutation graph and the corresponding matching diagram.

We call a temporal graph $\mathcal{G}=([n], \mathcal{E}, \tau)$ a temporal permutation graph if there exist $\tau$ permutations $\pi_{1}, \ldots, \pi_{\tau}$ of the vertices such that, for any two vertices $v<w$, we have $(\{v, w\}, t) \in \mathcal{E}$ if and only if $\pi_{t}(w)<\pi_{t}(v)$.

One can visualize a permutation with a matching diagram [12]. A matching diagram for a given permutation graph $G=([n], E)$ with permutation $\pi$ can be constructed by drawing $n$ points on a horizontal line and another $n$ points on a parallel line below it. Then each vertex $i$ is represented by a straight line segment connecting the $i$-th point on the top line to the $\pi(i)$-th point on the bottom line. Observe that two vertices share an edge in $G$ if and only if their corresponding line segments cross in the matching diagram. Fig. 2 provides an example for a permutation graph and the matching diagram of its underlying permutation.

First, we prove in Section 3.1 that TEMPORAL $(s, z)$-SEPARATION on temporal permutation graphs is NP-complete. In Section 3.2, we use the Kendall tau distance [19] to measure the dissimilarity of the permutations. Recall that the Kendall tau distance is a metric that counts the number of pairwise disagreements between two total orderings; it is also known as "bubble sort distance" since it measures the number of swaps needed to transform one permutation into the other. We show that Temporal $(s, z)$-SEPARATION becomes fixed-parameter tractable when parameterized by the combined parameter "sum of Kendall tau distances between consecutive permutations" and the separator size $k$.

\subsection{Hardness Results}

As is the case for split graphs, the class of permutation graphs contains all graphs with only one edge. This means that TEMPORAL $(s, z)$-SEPARATION for temporal graphs in which every layer is a permutation graph is NP-hard [13]. We can also show that Temporal $(s, z)$-SePAration remains NP-hard when restricted to the temporal permutation graphs (note that this means that the vertex ordering is the same for all layers).

Theorem 3.1. Temporal $(s, z)$-SEPARATION on temporal permutation graphs is NP-complete.

Proof. The problem is clearly contained in NP. We present a polynomial-time reduction from 3-SAT [14] to an instance of TEMPoral $(s, z)$-SEPARATION on a temporal permutation graph. In 3-SAT we are given a Boolean formula in conjunctive normal form with at most three literals in a clause and are asked whether there exists a truth assignment that satisfies the formula.

The constructed temporal graph contains two vertices for every unique variable in the original 3-SAT instance, exactly one of which has to be included in the separator, representing the choice of assigning a Boolean value to that variable. It also contains three vertices for every clause in the 3-SAT instance, one vertex per literal. Of these, exactly two per clause must be included in the separator. For each literal, there exists a temporal $(s, z)$-path that consists of 
that literal's vertex and a variable vertex that is part of the separator if and only if the literal evaluates to true. Since we can only remove two of a clause's three vertices, one of its literals needs to evaluate to true in order to avoid any remaining temporal $(s, z)$-paths.

Let $\Omega:=\left\{x_{1}, x_{2}, \ldots, x_{n}\right\}$ be the set of variables of a given 3-SAT instance with the Boolean formula $\left(\ell_{1,1} \vee \ell_{1,2} \vee \ell_{1,3}\right) \wedge \ldots \wedge\left(\ell_{m, 1} \vee \ell_{m, 2} \vee \ell_{m, 3}\right)$. We can construct a TemporaL $(s, z)-$ Separation instance $\mathcal{I}:=(\mathcal{G}=(V, \mathcal{E}, n+5 m+2), s, z, k=n+2 m)$, where $V=[2 n+3 m+2]$. For the sake of readability, we denote the vertices as follows:

$$
\begin{aligned}
s & :=1 & & \\
t_{i} & :=2 i & & \forall i \in[n] \\
f_{i} & :=2 i+1 & & \forall i \in[n] \\
c_{i, j} & :=1+2 n+3(i-1)+j & & \forall i \in[m], j \in[3] \\
z & :=2 n+3 m+2 & &
\end{aligned}
$$

We define the time-edges as

$$
\begin{aligned}
E_{i}(\mathcal{G}):= & \left\{\left\{t_{i}, v\right\},\left\{f_{i}, w\right\} \mid v, w \in V, t_{i}<v, w<f_{i}\right\}, \\
& \text { for } i \in \mathbb{N}, i \leq n, \\
E_{n+1}(\mathcal{G}):=\left\{\left\{s, t_{i}\right\},\left\{s, f_{i}\right\} \mid i \in \mathbb{N}, i \leq n\right\}, & \left\{\left\{, c_{i, j}\right\}\right\} \cup \\
E_{n+1+3(i-1)+j}(\mathcal{G}):= & \left\{\{v, w\} \mid w \in V, v<w \leq f_{n}\right\} \cup \\
& \left\{\left\{c_{i, j}, w\right\} \mid w \in V, c_{1,1} \leq w<c_{i, j}\right\}, \\
& \text { where } v=t_{k} \Leftrightarrow l_{i, j}=x_{k} \text { and } v=f_{k} \Leftrightarrow l_{i, j}=\neg x_{k}, \\
& \text { for } i, j \in \mathbb{N}, i \leq m, j \leq 3, \\
E_{n+3 m+2}(\mathcal{G}):= & \left\{\left\{z, c_{i, j}\right\} \mid i, j \in \mathbb{N}, i \leq m, j \leq 3\right\}, \\
E_{n+3 m+2 i+1}(\mathcal{G}):= & \left\{\left\{c_{i, 2}, v\right\},\left\{c_{i, 3}, w\right\} \mid v, w \in V, c_{i, 2}<v, w<c_{i, 3}\right\}, \\
& \text { for } i \in \mathbb{N}, i \leq m, \\
E_{n+3 m+2 i+2}(\mathcal{G}):= & \left\{\left\{c_{i, 1}, v\right\},\left\{c_{i, 2}, w\right\},\left\{c_{i, 3}, w\right\} \mid v, w \in V, c_{i, 1}<v, w<c_{i, 2}\right\}, \\
& \text { for } i \in \mathbb{N}, i \leq m .
\end{aligned}
$$

We first show that we can give a permutation for every layer that produces the corresponding edge set.

In layers 1 through $n$ and all layers $n+3 m+2 i+1, i \in \mathbb{N}$, there is one vertex with edges to all smaller vertices and one with edges to all larger vertices. The corresponding permutation maps these vertices to the first respectively last position while keeping the relative order of all other vertices unchanged. If said two vertices are $i$ and $i+1$, then the corresponding permutation would be $(2,3, \ldots, i,|V|, 1, i+1, \ldots,|V|-1)$. See also Fig. 3 and Fig. 7 .

In layer $n+1$, vertex $s$ has edges to the set $\left\{2,3, \ldots, f_{n}\right\}$, yielding the permutation $\left(f_{n}, 1,2, \ldots\right.$, $\left.f_{n}-1, f_{n}+1, \ldots,|V|\right)$. For layer $n+3 m+2$, the situation is similar. See also Fig. 4 and Fig. 6 .

In each of the layers $n+2$ through $n+3 m+1$ there is a vertex $v \leq 2 n+1$ and a vertex $c>$ $2 n+1$. Each of these two vertices has an edge to every other vertex lying between themselves and $f_{n}+1 / 2$. This corresponds to the permutation $\left(1,2, \ldots, v-1, f_{n}+1, v, \ldots, f_{n}-1, f_{n}+\right.$ $\left.2, \ldots, c, f_{n}, c+1, \ldots,|V|\right)$. See Fig. 5 .

In all layers $n+3 m+2 i+2, i \in \mathbb{N}$, there are two consecutive vertices with edges to all vertices below them but no edge to each other, and one vertex with edges to all vertices above it. This corresponds to the positions of the former two vertices having the first two positions in the permutation and the latter vertex having the last position in the permutation while the 


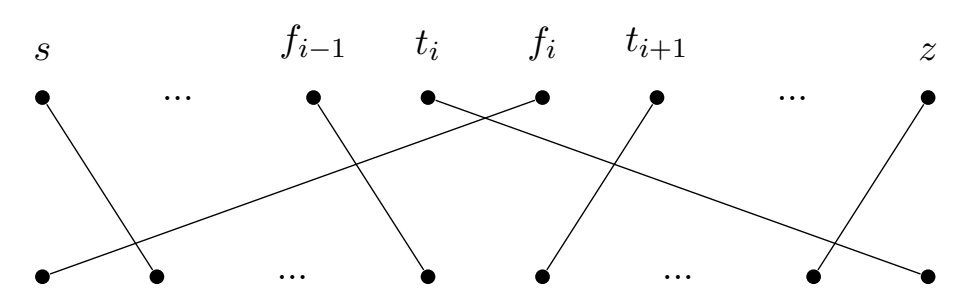

Figure 3: Matching diagram for some layer $i \in \mathbb{N}, 1<i<n$, of $\mathcal{G}$ in the proof of Theorem 3.1.

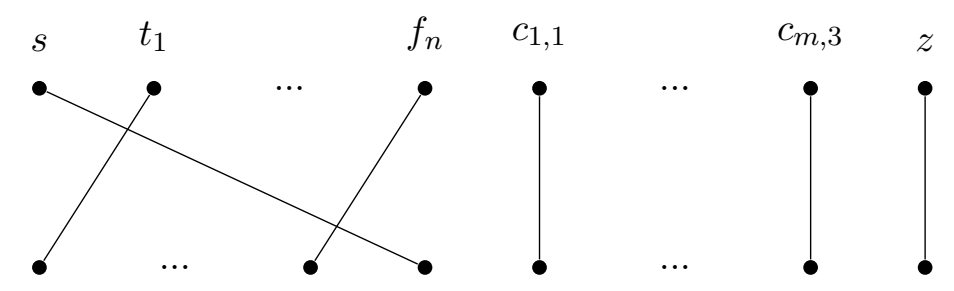

Figure 4: Matching diagram for layer $n+1$ of $\mathcal{G}$ in the proof of Theorem 3.1.

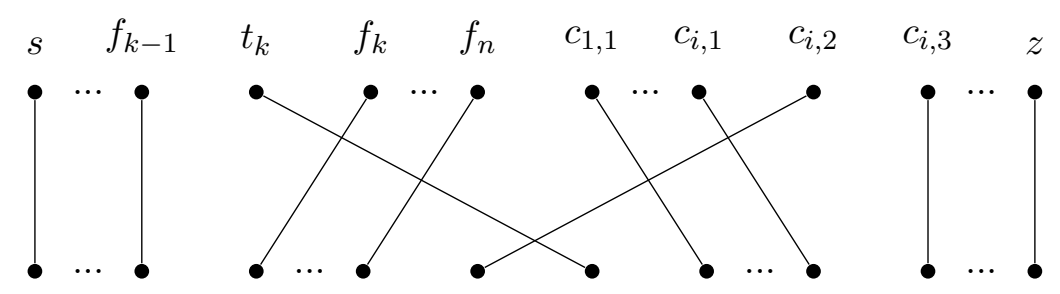

Figure 5: Matching diagram for layer $n+1+3(i-1)+2, i \in \mathbb{N}, 1<i<m$, of $\mathcal{G}$ in the proof of Theorem 3.1, assuming that $l_{i, 2}=x_{k}$.

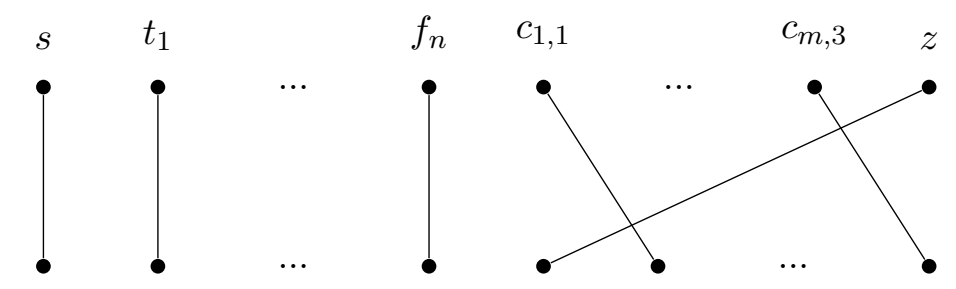

Figure 6: Matching diagram for layer $n+3 m+2$ of $\mathcal{G}$ in the proof of Theorem 3.1.

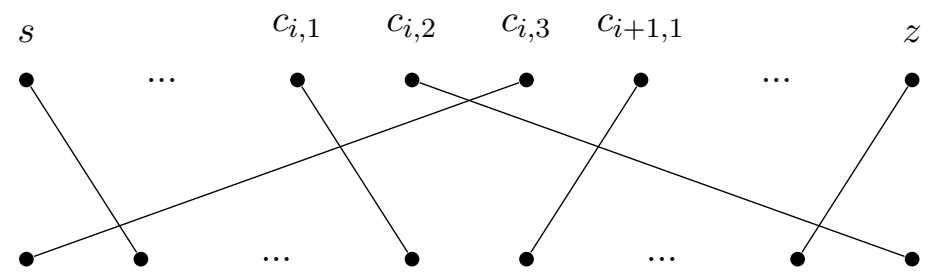

Figure 7: Matching diagram for some layer $n+3 m+2 i+1, i \in \mathbb{N}, 1<i<m$, of $\mathcal{G}$ in the proof of Theorem 3.1. 


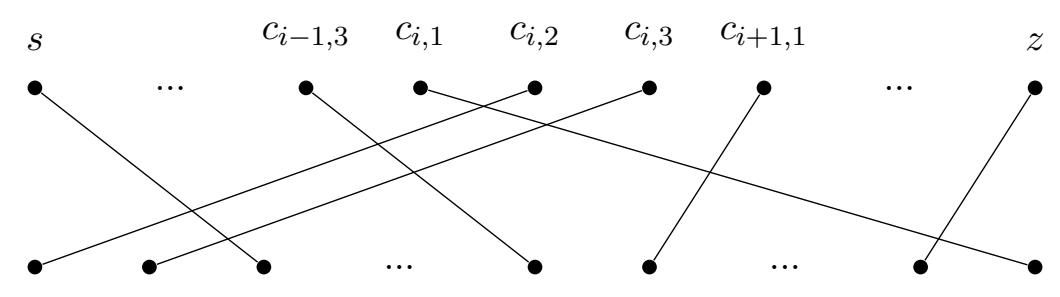

Figure 8: Matching diagram for some layer $n+3 m+2 i+2, i \in \mathbb{N}, 1<i<m$, of $\mathcal{G}$ in the proof of Theorem 3.1 .

order of all other elements remains unchanged. For example, for layer $n+3 m+2 i+2$, this yields the permutation $\left(3,4, \ldots, c_{i, 2},|V|, 1,2, c_{i, 3}, \ldots,|V|-1\right)$. See Fig. 8.

Correctness. We prove that the Boolean formula is satisfiable if and only if $\mathcal{I}$ is a yes-instance.

$\Leftarrow$ : For every $i \in \mathbb{N}, i \leq n$, there exists an $(s, z)$-path through $t_{i}$ and $f_{i}$ in layer $i$, meaning that one of these two vertices has to be included in any temporal $(s, z)$-separator. For every $i \in$ $\mathbb{N}, i \leq m$, there is an $(s, z)$-path through $c_{i, 2}$ and $c_{i, 3}$ in layer $n+3 m+2 i+1$ and two paths in layer $n+3 m+2 i+2$, first going through $c_{i, 1}$ and then either $c_{i, 2}$ or $c_{i, 3}$. To remove all of these paths any two of the three vertices have to be in any temporal $(s, z)$-separator.

To satisfy all of these requirements, a temporal $(s, z)$-separator needs to contain at least $n+$ $2 m$ vertices. Since this equals $k$, a separator that is a solution to $\mathcal{I}$ cannot contain any more vertices than required above. Specifically, for each $i \in \mathbb{N}, i \leq n$, only one of the two vertices $t_{i}$ and $f_{i}$ can be in the separator, and for every clause $\left(\ell_{i, 1} \vee \ell_{i, 2} \vee \ell_{i, 3}\right)$ in the Boolean formula, there exists one vertex $c_{i, j}, j \in\{1,2,3\}$ that is not in the separator. If $\ell_{i, j}$ is of the form $x_{k}$, then setting $x_{k}$ to true satisfies the clause and there exists an $(s, z)$-path through $t_{k}$ and $c_{i, j}$ spanning layer $n+1$, layer $n+1+3(i-1)+j$, and layer $n+3 m+2$, meaning that $t_{k}$ must be in the separator. If $\ell_{i, j}$ is of the form $\neg x_{k}$, then setting $x_{k}$ to false satisfies the clause and there exists an $(s, z)$-path through $f_{k}$ and $c_{i, j}$ in those three layers, meaning that $f_{k}$ must be in the separator. Hence, if we set every variable $v_{k}$ in $\Omega$ to true if the separator contains $t_{k}$, and to false if the separator contains $f_{k}$, all clauses, and therefore the Boolean formula as a whole, are satisfied.

$\Rightarrow$ : Given an interpretation of $\Omega$ that satisfies the Boolean formula, we can construct a temporal $(s, z)$-separator $S$ of size $k$ for $\mathcal{G}$. For $i \in \mathbb{N}, i \leq n$, if the interpretation sets $x_{i}$ to true, then we include $t_{i}$ in the separator, if the interpretation set $x_{i}$ to false, then we include $f_{i}$ in the separator. Since the interpretation satisfies the Boolean formula, in every clause $\left(\ell_{i, 1} \vee \ell_{i, 2} \vee \ell_{i, 3}\right)$ in the formula at least one literal has to evaluate to true. We choose $\ell_{i, j}$ to be such a literal and then include the vertices in $\left\{c_{i, k} \in V \mid k \neq j\right\}$ in the separator. After doing this for each clause, we now have a temporal $(s, z)$-separator of size $k$.

In the following we will refer to the vertex set $\left\{v \in V \mid v \leq f_{n}\right\}$ as $V_{v}$, and to $\left\{v \in V \mid c_{1,1} \leq\right.$ $v\}$ as $V_{c}$. In each layer $i$ for $i \in \mathbb{N}, i \leq n$, only vertices up to $f_{i}$ are reachable from $s$ without having to pass through both $t_{i}$ and $f_{i}$, so by layer $n$, only vertices in $V_{v}$ can be reached. In layer $n+1$, there are no edges leading to $V_{c}$.

In each layer $n+1+3(i-1)+j$ for $i, j \in \mathbb{N}, i \leq m, j \leq 3$, the only edge connecting $V_{v}$ and $V_{c}$ is $\left\{v, c_{i, j}\right\}$ where $v=t_{k} \Leftrightarrow \ell_{i, j}=x_{k}$ and $v=f_{k} \Leftrightarrow \ell_{i, j}=\neg x_{k}$, with $k \in \mathbb{N}, k \leq n$. We look at two cases:

- If $c_{i, j}$ is in $S$, then $V_{c}$ cannot be reached in this layer.

- If we did not include $c_{i, j}$ in $S$, then $\ell_{i, j}$ must evaluate to true, now we distinguish two cases again: 
- If $\ell_{i, j}=x_{k}$, then $x_{k}$ must be set to true and $v=t_{k} \in S$.

- If $\ell_{i, j}=\neg x_{k}$, then $x_{k}$ must be set to false and $v=f_{k} \in S$.

Therefore, in none of these layers any vertex in $V_{c}$ is reachable from $V_{v}$.

In layer $n+3 m+2$, there are no edges leading to $V_{c}$. In the layers $n+3 m+2 i+1$ and $n+3 m+2 i+2$ for each $i \in \mathbb{N}, i \leq m$, no vertices greater than $c_{i, 3}$ can be reached from $V_{v}$ without passing through at least two of the vertices $c_{i, 1}, c_{i, 2}$, and $c_{i, 3}$. Since only one of these was not included in $S$, this is not possible.

Hence, by layer $n+5 m+2$, which is the last layer, no vertex greater than $2 n+3 n+1$ can be reached from $s$. Since $z=|V|=2 n+3 m+2$, there remains no temporal $(s, z)$-path and $S$ is indeed a temporal $(s, z)$-separator.

We remark that the reduction we use to obtain Theorem 3.1 uses a unbounded number of time steps and also the maximum Kendall tau distance between any two consecutive permutations is unbounded. However, by introducing additional layers, one can decrease the Kendall tau distance between any two consecutive layers to one. The main idea is to gradually change a layer to an independent set and then gradually to the next layer. This can be done in a way that does not introduce any new temporal paths.

\subsection{Fixed-Parameter Tractability Results}

In this section, we examine the effect of limiting how much the permutations of the layers of the temporal permutation graph change. We can do so by measuring the Kendall tau distance between the permutations of consecutive layers.

We will show that TEMPORAL $(s, z)$-SEPARATION on temporal permutation graphs is fixedparameter tractable with respect to the sum of Kendall tau distances between all pairs of consecutive permutations plus separator size $k$. For this we need to demonstrate that these parameters limit the number of $(s, z)$-separators that a layer does not have in common with another layer.

First, we introduce the concept of scanlines. A scanline is any line segment in the matching diagram of a permutation with one end on each horizontal line. If $s$ lies on one side of the scanline and $z$ on the other, then the set of all vertices whose line segments cross the scanline is an $(s, z)$-separator. We call such a separator a scanline separator. Bodlaender et al. [3] have shown that every minimal separator in a permutation graph is a scanline separator and that there are at most $(n-1)(2 k+3)$ scanline separators of size at most $k$ [3, Proof of Lemma 3.6], where $n$ is the number of vertices.

Lemma 3.2. Let $G_{1}=\left([n], E_{1}\right)$ and $G_{2}=\left([n], E_{2}\right)$ be two permutation graphs. If the two corresponding permutations have Kendall tau distance $d$, then the number of scanline separators of size at most $k$ in $G_{2}$ that are not also scanline separators in $G_{1}$ is at most $d \cdot(2 k+1)$.

Proof. We begin by showing that for every endpoint on the bottom line, there are at most $2 k+1$ separators of size at most $k$ defined by a scanline with that endpoint. For a given bottom endpoint, we look at the leftmost endpoint on the top line that defines such a separator. Moving right, every time we pass an element of the permutation, its line is added to those crossed by the scanline if it was completely to the right of it before, and removed from them if it crossed the scanline before. This corresponds to adding or removing one vertex from the defined separator.

Since our scanline can pass every element only once, the vertices that were added in this process will not be removed again. If we have already passed more than $2 k$ points on the top line, then more than $k$ of these represent vertices which were not present in the initial separator and were thus added. Therefore the resulting separator contains more than $k$ vertices. Therefore, 
all separators of size at most $k$ must be produced in the first $2 k$ steps of this process. Together with the separator defined by the initial scanline, this gives us at most $2 k+1$ scanline separators as claimed.

Upon moving from $G_{1}$ to $G_{2}$, the set of lines crossing any given scanline only changes, if any of the $d$ swaps swapped the two points immediately to the left and right of the lower end of the scanline. By the above, this means at most $d \cdot(2 k+1)$ many scanlines produce different separators in $G_{1}$ and $G_{2}$. Hence, the number of new scanline separators in $G_{2}$ does not exceed $d \cdot(2 k+1)$.

Now we show that reachability in temporal permutation graphs follows the vertex order: If a vertex reaches another vertex, then it also reaches all vertices in between.

Lemma 3.3. Let $G=([n], E)$ be a permutation graph and $v, w, x \in[n]$ three vertices with $v<w<x$. If there exists a path from $v$ to $x$, then there also exist paths from $w$ to both, $v$ and $x$.

Proof. Let $\pi$ be the corresponding permutation. Since $v$ and $x$ are connected, it suffices to show that there is a path from one of them to $w$. We first look at the case that there is an edge between $v$ and $x$. This means that $\pi(v)>\pi(x)$. If $w$ does not have an edge to either $v$ or $x$, then neither the positions of $v$ and $w$ nor $w$ and $x$ get reversed by $\pi$, so $\pi(v)<\pi(w)<\pi(x) \Rightarrow$ $\pi(v)<\pi(x)$, a contradiction. Therefore, either $v$ or $x$ must be connected to $v$ via a single edge.

Now we use induction to show that the lemma holds for a $(v, x)$-path of any length. A $(v, x)$ path of length 1 is the same as an edge between the two vertices. The induction hypothesis is that if there exists a $(v, x)$-path of length $\ell \in \mathbb{N}$, then there exist paths to $w$ from $v$ and $x$.

We show that if there exists a $(v, x)$-path $p$ of length $\ell+1$, then there exist paths to $w$ from $v$ and $x$. Let $v^{\prime}$ be the element of $p$ that follows $v$. If $w<v^{\prime}$, then, since $v<w<v^{\prime}$ and there exists an edge between $v$ and $v^{\prime}$, there is either an edge between $v$ and $w$, or an edge between $v^{\prime}$ and $w$, forming a path from $v$ to $w$. If $v^{\prime}<w$ then, since $v^{\prime}<w<x$ and there exists a $\left(v^{\prime}, x\right)$-path of length $\ell$, due to the induction hypothesis, there exists a path from $x$ to $w$.

We now present a parameterized algorithm for TEMPORAL $(s, z)$-SEPARATION on a temporal permutation graph $\mathcal{G}$. For this, we introduce the parameter $d_{\Sigma}:=\sum_{t=1}^{\tau-1} d_{\mathrm{Kt}}\left(\pi_{t}, \pi_{t+1}\right)$, where $d_{\mathrm{Kt}}$ denotes the Kendall tau distance and $\pi_{t}$ is the $t$-th permutation of $\mathcal{G}$. Note that taking the maximum instead of the sum does not provide a helpful parameter since the hardness reduction we used to obtain Theorem 3.1 can be modified such that the Kendall tau distance of any two consecutive layers is one.

Theorem 3.4. For a temporal permutation graph, TEMPORAL $(s, z)$-SEPARATION can be solved in $\mathcal{O}\left(\left(d_{\Sigma}(2 k+1)\right)^{k} n \cdot|\mathcal{E}|+\tau n^{2}\right)$ time.

Proof. We present an algorithm that runs in $\mathcal{O}\left(\left(d_{\Sigma}(2 k+1)\right)^{k} n \cdot|\mathcal{E}|+\tau n^{2}\right)$ time, which determines whether a given Temporal $(s, z)$-Separation-instance has a solution (see Algorithm 1 ).

Remember that the total number of scanline separators of size at most $k$ in layer 1 is at most $(n-1)(2 k+3)$. Furthermore, in all layers after layer 1, the number of minimal (i.e., scanline) separators which are not shared with the previous layer is at most $d_{\Sigma}(2 k+1)$ (see Lemma 3.2). Hence the first call of GetSeparator iterates at most $(n-1)(2 k+3)+d_{\Sigma}(2 k+1) \in$ $\mathcal{O}\left(d_{\Sigma}(2 k+1) \cdot n\right)$ times and every recursive call iterates at most $d_{\Sigma}(2 k+1)$ times.

Due to the condition in Line 14, every time a recursive call is made, the set passed to $S$ contains at least one vertex more than before, but never exceeds the size $k$. Thus the maximum recursion depth is $k$. In every call of GetSeparator it is checked in $\mathcal{O}(|E|)$ time whether $S$ 


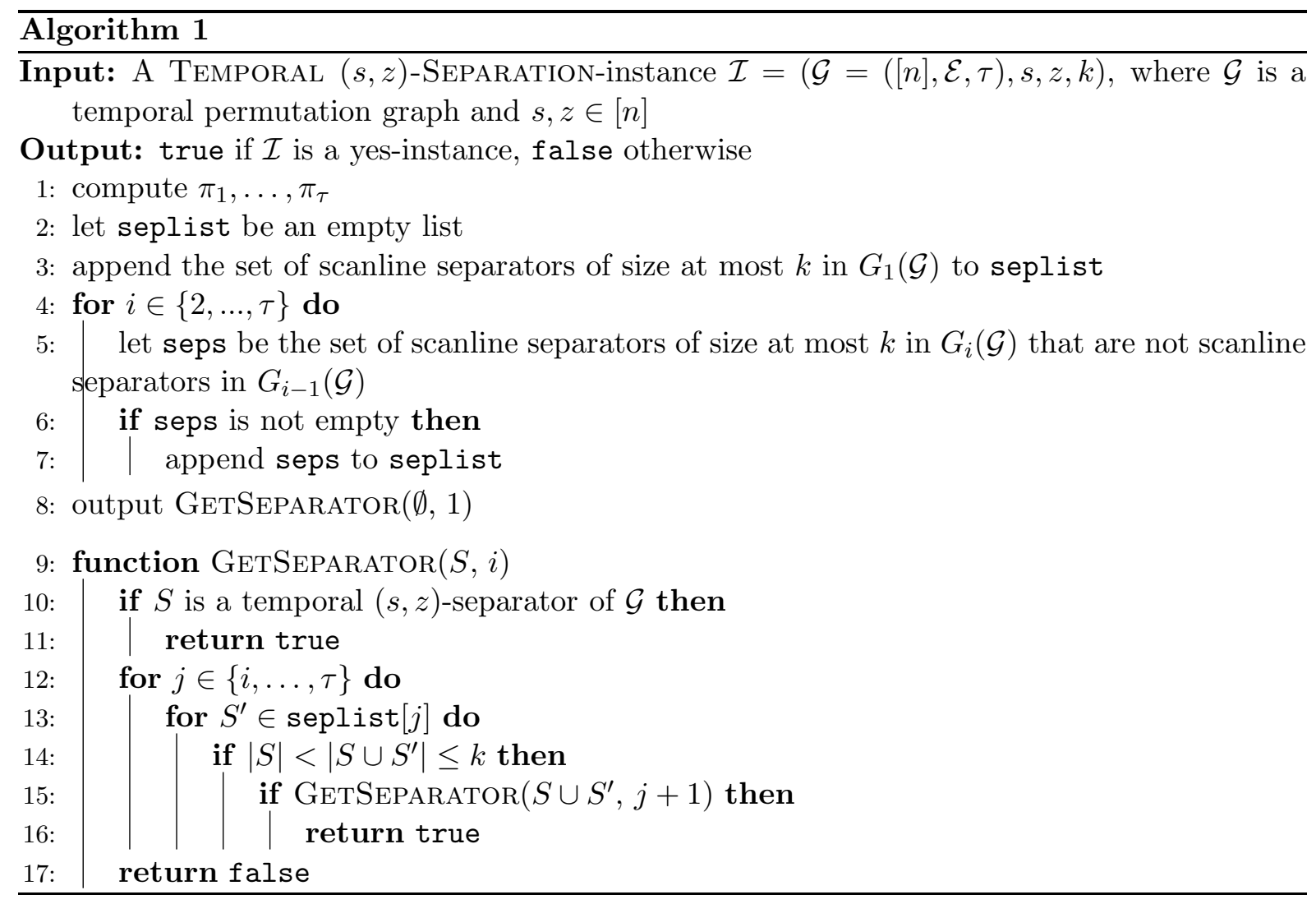

is a temporal $(s, z)$-separator. This results in a running time of $\mathcal{O}\left(\left(d_{\Sigma}(2 k+1)\right)^{k} n \cdot|\mathcal{E}|\right)$ for the initial call of GETSEPARATOR.

It remains to show that Lines 1 through 7 can be performed in $\mathcal{O}\left(\tau n^{2}\right)$ time. To construct some permutation $\pi_{i}$, we first iterate once through the edges of $G_{i}$ and build, for each vertex $v \in[n]$, the set $I(v):=\left\{w \in[n] \mid w<v\right.$ and $\left.\{v, w\} \in E\left(G_{i}\right)\right\}$. Then we can incrementally construct $\pi_{i}$ from an empty tuple by going through all vertices in ascending order and inserting each vertex $v$ exactly to the left of all elements of $I(v)$. If $I(v)$ is implemented using a hash set, this takes $\mathcal{O}\left(n^{2}\right)$ time for each layer. Afterwards, building seplist again takes $\mathcal{O}\left(n^{2}\right)$ time for each layer as there are $n^{2}$ potential scanlines and each only requires constant checking time if they are all iterated in order.

Correctness. It is easy to see that Algorithm 1 will never output true when $\mathcal{I}$ is a noinstance, as the condition in Line 10 can only evaluate to true if there exists a temporal $(s, z)$ separator.

It remains to be shown that Algorithm 1 will always output true when $\mathcal{I}$ is a yes-instance. Without loss of generality we assume that $s<z$. We also assume that there exists a minimal temporal $(s, z)$-separator $S^{*}$ of size at most $k$ in $\mathcal{G}$. Due to Lemma 3.3, every layer $t$ in $\mathcal{G}-S^{*}$ has some farthest reachable vertex $f_{t}$ between $s$ and $z$ such that until time $t, s$ can reach all vertices $v$ with $s \leq v \leq f_{t}$ via temporal paths but no vertex $v$ with $v>f_{t}$. Clearly $f_{t} \leq f_{t+1}$. This means that in each layer $t, S^{*}$ must contain a scanline separator that separates $f_{t}$ from all vertices $v>f_{t}$. We denote this scanline separator by $S_{t}^{*}$. By minimality of $S^{*}, S^{*}=\bigcup_{t=1}^{\tau} S_{t}^{*}$.

Trivially, the following property holds for the initial call of GetSeparator:

$$
\bigcup_{t=1}^{i-1} S_{t}^{*} \subseteq S \subseteq S^{*}
$$


We next show that whenever $(*)$ holds for a call of GetSeparator, then either $S=S^{*}$ or $(*)$ also holds for some recursive call. This implies that some recursive call will eventually produce $S^{*}$.

So assume now $(*)$ holds with $S \neq S^{*}$. Since $S$ is then not a temporal $(s, z)$-separator, $\operatorname{GetSeparator}(S, i)$ iterates through all scanline separators in seplist $[i]$ through seplist $[\tau]$, one of which must be the first scanline separator $S_{t}^{*}$ which is not already contained in $S$. When it gets to that separator it makes a recursive call $\operatorname{GetSeparator}\left(S \cup S_{t}^{*}, i+1\right)$. This recursive call then again satisfies $(*)$.

We leave open whether Temporal $(s, z)$-SEPARATION is fixed-parameter tractable or becomes W[1]-hard when parameterized by either only the separator size $k$ or only the sum $d_{\Sigma}$ of Kendall tau distances of permutations of consecutive layers.

\section{Conclusion}

We showed that TEMPoral $(s, z)$-SEPARATION remains NP-complete on temporal split graphs even when there are only $\tau \geq 4$ layers, but it becomes fixed-parameter tractable when parameterized by the lifetime $\tau$ combined with the number $p$ of "switching vertices", that is, vertices that switch between the independent set and the clique. We leave open, however, whether one can obtain fixed-parameter tractability when only parameterizing by $p$. Another natural restriction we can place on temporal split graphs is limiting the size of the independent set for all layers. We conjecture that TEMPORAL $(s, z)$-SEPARATION is fixed-parameter tractable with respect to the maximum size of the independent set.

We also showed that TEMPORAL $(s, z)$-SEPARATION remains NP-complete on temporal permutation graphs, but becomes fixed-parameter tractable with respect to the separator size $k$ plus the sum $d_{\Sigma}$ of Kendall tau distances of permutations of consecutive layers. We left open the complexity of Temporal $(s, z)$-SEPARATION on temporal permutation graphs when parameterized by the lifetime $\tau$. Whether the problem stays fixed-parameter tractable or becomes $\mathrm{W}[1]$-hard when parameterized by either only the separator size $k$ or only the sum $d_{\Sigma}$ of Kendall tau distances of permutations of consecutive layers remains open as well.

Lastly, we leave for future research whether our results also hold in the strict case, that is, when the temporal paths that are to be destroyed by the separator have strictly increasing time labels. Most of our algorithms heavily rely on the fact that temporal paths may use several time edges with the same label, and hence they can presumably not be adapted to the strict setting in a straightforward way.

\section{References}

[1] E. A. Bender, L. B. Richmond, and N. C. Wormald. Almost all chordal graphs split. Journal of the Australian Mathematical Society. Series A. Pure Mathematics and Statistics, 38(2): 214-221, 1985. doi: 10.1017/S1446788700023077. 3

[2] M. Bentert, A.-S. Himmel, A. Nichterlein, and R. Niedermeier. Efficient computation of optimal temporal walks under waiting-time constraints. Applied Network Science, 5(1):73, 2020. doi: 10.1007/s41109-020-00311-0. 2

[3] H. L. Bodlaender, T. Kloks, and D. Kratsch. Treewidth and pathwidth of permutation graphs. SIAM Journal on Discrete Mathematics, 8:606-616, 1995. doi: 10.1137/ S089548019223992X. 14 
[4] S. P. Borgatti and M. G. Everett. Models of core/periphery structures. Social Networks, 21(4):375-395, 2000. doi: 10.1016/S0378-8733(99)00019-2. 2, 3

[5] A. Brandstädt, V. B. Le, and J. P. Spinrad. Graph Classes - A Survey. Society for Industrial and Applied Mathematics, 1999. doi: 10.1137/1.9780898719796. 2

[6] B.-M. Bui-Xuan, A. Ferreira, and A. Jarry. Computing shortest, fastest, and foremost journeys in dynamic networks. International Journal of Foundations of Computer Science, 14(02):267-285, 2003. doi: 10.1142/S0129054103001728. 2

[7] S. Buß, H. Molter, R. Niedermeier, and M. Rymar. Algorithmic aspects of temporal betweenness. In Proceedings of the 26th SIGKDD Conference on Knowledge Discovery and Data Mining (KDD), pages 2084-2092, 2020. doi: 10.1145/3394486.3403259. 2

[8] J. Chen, I. A. Kanj, and G. Xia. Improved upper bounds for vertex cover. Theoretical Computer Science, 411(40-42):3736-3756, 2010. doi: 10.1016/j.tcs.2010.06.026. 8

[9] J. Enright, K. Meeks, G. B. Mertzios, and V. Zamaraev. Deleting edges to restrict the size of an epidemic in temporal networks. Journal of Computer and System Sciences, 119: 60-77, 2021. doi: 10.1016/j.jcss.2021.01.007. 2

[10] J. Enright, K. Meeks, and F. Skerman. Assigning times to minimise reachability in temporal graphs. Journal of Computer and System Sciences, 115:169-186, 2021. doi: 10.1016/j.jcss. 2020.08.001. 2

[11] T. Erlebach, M. Hoffmann, and F. Kammer. On temporal graph exploration. Journal of Computer and System Sciences, 119:1-18, 2021. doi: 10.1016/j.jcss.2021.01.005. 2

[12] S. Even, A. Pnueli, and A. Lempel. Permutation graphs and transitive graphs. Journal of the $A C M, 19(3): 400-410,1972$. doi: 10.1145/321707.321710. 3, 9, 10

[13] T. Fluschnik, H. Molter, R. Niedermeier, M. Renken, and P. Zschoche. Temporal graph classes: A view through temporal separators. Theoretical Computer Science, 806:197-218, 2020. doi: $10.1016 /$ j.tcs.2019.03.031. 2, 3, 10

[14] M. R. Garey and D. S. Johnson. Computers and Intractability: A Guide to the Theory of NP-Completeness. W. H. Freeman and Company, 1979. ISBN 978-0-7167-1045-5. 6, 10

[15] M. C. Golumbic. Algorithmic Graph Theory and Perfect Graphs. Elsevier, 2004. ISBN 978-0-444-51530-8. 2, 3, 9

[16] J. Guo, F. Hüffner, and R. Niedermeier. A structural view on parameterizing problems: Distance from triviality. In Proceedings of the 1st International Workshop on Parameterized and Exact Computation (IWPEC), pages 162-173, 2004. doi: 10.1007/978-3-540-28639-4 \ -15. 3

[17] P. Heggernes and F. Mancini. Minimal split completions of graphs. In Proceedings of the 7th Latin American Symposium on Theoretical Informatics (LATIN), volume 3887, pages 592-604, 2006. doi: 10.1007/11682462\_55. 7

[18] D. Kempe, J. Kleinberg, and A. Kumar. Connectivity and inference problems for temporal networks. Journal of Computer and System Sciences, 64(4):820-842, 2002. doi: 10.1006/ jcss.2002.1829. 1, 2 
[19] M. G. Kendall. A new measure of rank correlation. Biometrika, 30(1/2):81-93, 1938. doi: 10.2307/2332226. 3, 10

[20] G. B. Mertzios, O. Michail, and P. G. Spirakis. Temporal network optimization subject to connectivity constraints. Algorithmica, 81(4):1416-1449, 2019. doi: 10.1007/ s00453-018-0478-6. 2

[21] R. Niedermeier. Invitation to Fixed-Parameter Algorithms. Oxford University Press, 2006. doi: 10.1093/ACPROF:OSO/9780198566076.001.0001. 3

[22] A. Sen, H. Deng, and S. Guha. On a graph partition problem with application to VLSI layout. Information Processing Letters, 43(2):87-94, 1992. doi: 10.1016/0020-0190(92) 90017-P. 3

[23] P. Zschoche, T. Fluschnik, H. Molter, and R. Niedermeier. The complexity of finding small separators in temporal graphs. Journal of Computer and System Sciences, 107:72-92, 2020. doi: 10.1016/j.jcss.2019.07.006. 2, 4 